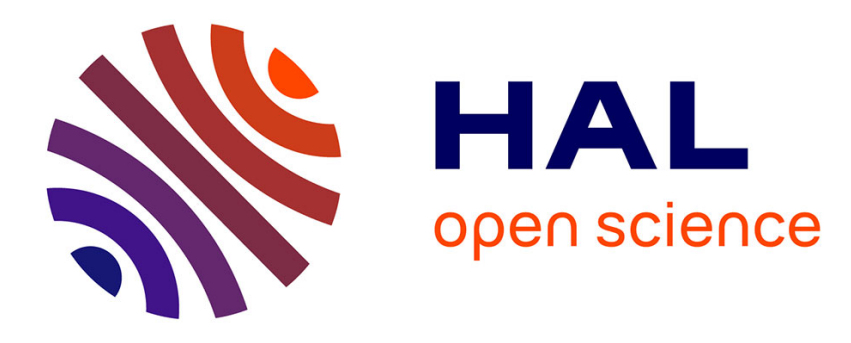

\title{
Slow relaxation processes in supercooled liquids
}

L. Sjögren

\section{To cite this version:}

L. Sjögren. Slow relaxation processes in supercooled liquids. Journal de Physique IV Proceedings, 1993, 03 (C1), pp.C1-117-C1-128. 10.1051/jp4:1993111 . jpa-00251554

\section{HAL Id: jpa-00251554 https://hal.science/jpa-00251554}

Submitted on 1 Jan 1993

HAL is a multi-disciplinary open access archive for the deposit and dissemination of scientific research documents, whether they are published or not. The documents may come from teaching and research institutions in France or abroad, or from public or private research centers.
L'archive ouverte pluridisciplinaire HAL, est destinée au dépôt et à la diffusion de documents scientifiques de niveau recherche, publiés ou non, émanant des établissements d'enseignement et de recherche français ou étrangers, des laboratoires publics ou privés. 


\section{Slow relaxation processes in supercooled liquids}

\section{SJÖGREN}

Institute of Theoretical Physics, Chalmers University of Technology, 41296 Göteborg, Sweden

The properties of the $\alpha$ - and $\beta$-processes as described within the mode-coupling theory for the dynamics of supercooled liquids are reviewed. These slow relaxation processes are ruled by generic properties of underlying bifurcation singularities in the nonlinear equations of motion. The singularities belong to the cuspoid family $A_{k}$ with $k \geq 2$, and lead to various scaling laws for the dynamics.

Les propriétés des processus $\alpha$ et $\beta$, comme décrites dans la théorie de "mode-coupling" pour la dynamique des liquides super-réfrigérées, sont résumées. Ces prosessus lents de rélaxation sont determinés par les propriétes génériques des singularités de bifurcation dans les équations non-linéaires de mouvement. Les singularités sont dans la famille cuspoide $A_{k}$ avec $k \geq 2$, et mène à plusieurs lois d'échelle pour la dynamique.

\section{Introduction}

When a liquid is supercooled below the melting point $T_{\mathrm{m}}$ structural rearrangements of the atoms or molecules becomes increasingly more difficult. This leads to a rapid slowing down of the relaxation processes, and the appearence of low frequency peaks in various susceptibility spectra, the so called $\alpha$-peaks. The position $\omega_{\max }$ of the spectral maximum defines a time scale $\tau_{\alpha}=2 \pi / \omega_{\max }$ for the relaxation process. A transition to a glassy state occurs when the structural relaxation time $\tau_{\alpha}$ becomes of the same order as the experimental time-scale, so that structural rearrangements no longer can be observed. Conventionally the glass transition temperature $T_{\mathrm{g}}$ is defined as the temperature where the shear viscosity $\eta \propto 1 / \tau_{\alpha}$ reaches about $10^{13}$ poise.

Susceptibility spectra of supercooled liquids quite often exhibit additional resonances that are located at a frequency above the $\alpha$-peaks, but below the band of typical microscopic excitations. In many cases one can relate these resonances to reorientational motions of molecules or of side groups of the constituents. However such resonances appear also in simple glass formers, where they cannot be attributed to specialities of the microscopic structure. It was argued by Goldstein and Johari [1] and Johari [2] that these resonances referred to as $\beta$-peaks are genuine features of the glass transition, and reflects the motion of mobile clusters in a frozen environment.

The $\alpha$ - and $\beta$-processes can be observed by various experimental techniques like dielectric- and mechanical relaxation measurements or neutron and light scattering. From these measurements one can extract valuable information about their temperature and frequency dependencies. An outstanding feature of $\alpha$ - as well as $\beta$-relaxation is stretching of the dynamics over frequencywindows which are several decades wide. Recent mode-coupling theories (MCT) [3, 4] (see also [5] and [6] for recent reviews) make detailed predictions for many properties of the $\beta$ - and $\alpha$-processes. Here some of these results will be summarised. 


\section{Basic theory}

The rapid increase of the structural relaxation time $\tau_{\alpha}$ near $T_{\mathrm{g}}$ implies that rearrangements of the atoms between various configurations becomes slower and slower upon supercooling. These rearrangements can be expressed as fluctuations in the local density of the system. Detailed information about the time-dependence of structural rearrangements is therefore contained in the density-density correlation function:

$$
\phi_{q}(t)=\left\langle\delta \rho(\boldsymbol{q}, t) \delta \rho^{*}(\boldsymbol{q}, 0)\right\rangle / S_{q} .
$$

Here $\rho(\boldsymbol{q} . t)$ denotes a microscopic density fluctuation of wavevector $\boldsymbol{q}$ and $S_{q}=\langle| \delta \rho\left(\left.\boldsymbol{q}\right|^{2}\right\rangle$ denotes the static structure factor. The density correlation function can be expressed in terms of a generalized longitudinal viscosity $M_{q}(z)$

$$
\phi_{q}(z)=-1 /\left[z-\Omega_{q}^{2} /\left[z+M_{q}(z)\right]\right] .
$$

where $\phi_{q}(z)$ denotes the Laplace-transform

$$
\phi_{q}(z)=\mathrm{i} \int_{0}^{\infty} \mathrm{d} t \mathrm{e}^{\mathrm{i} z t} \phi_{q}(t)
$$

For real $z=\omega(3)$ defines a reactive part $\phi_{q}^{\prime}(\omega)$ and a loss spectrum $\phi_{q}^{\prime \prime}(\omega)$. The latter function is of central importance for experiments, since the dynamical structure factor $S(q, \omega)=S_{q} \phi_{q}^{\prime \prime}(\omega)$ determine the cross section for neutron- and light scattering measurements, and the corresponding susceptibility $\chi_{q}^{\prime \prime}(\omega) \propto \omega \phi_{q}^{\prime \prime}(\omega)$ is obtained in loss measurements.

The characteristic frequency $\Omega_{q}=\left(q^{2} / \beta m S_{q}\right)^{1 / 2}$, with $\beta=1 / k_{\mathrm{B}} T$, defines the scale for the microscopic dynamics. It is of the same order $10^{-13}-10^{-14} \mathrm{~s}^{-1}$ as in the corresponding crystalline solids. The generalised viscosity can be calculated within a kinetic theory or field theoretic approach [7-9]. The resut is:

$$
M_{q}(z)=\Omega_{q}^{2}\left[\nu_{q}(z)+m_{q}(z)\right] /\left\{1-\delta_{q}(z)\left[\nu_{q}(z)+m_{q}(z)\right]\right\}
$$

Here $\nu_{q}(z)$ describes the details of the liquid dynamics on the scale $\Omega_{q}$. This part reflects essentially short ranged binary uncorrelated collisions. For low frequencies of interest here it provides just a white noise spectral background, $\nu_{q}(z)=\mathrm{i} \nu_{q}$. The nontrivial parts of the relaxation kernel are the terms $m_{q}(z)$ and $\delta_{q}(z)$. The former part represent the cooperative motions of any particle and its surrounding. In a dense liquid this motion is dominated by the so called cage effect, where any atom is to some extent trapped by the surrounding atoms for some time. When the liquid is supercooled this trapping mechanism becomes stronger and may lead to a localization of a cluster of atoms for a long time. The resulting increase of the viscosity $M_{q}(z)$ leads to a slowing down of density fluctuations, and therefore to an additional enhancement of the cage effect. So there is an inherent feedback mechanism which tend to slow down particle rearrangements.

The cage effect is accounted for by products of density correlation functions. The resulting equation for the viscosity reads

$$
m_{q}(t)=\mathcal{F}_{q}\left(\boldsymbol{V}, \phi_{k}(t)\right)
$$

where,

$$
\mathcal{F}_{\boldsymbol{q}}\left(\boldsymbol{V}, f_{k}\right)=\frac{1}{2} \sum_{k, \boldsymbol{p}} V(\boldsymbol{q}, \boldsymbol{k}, \boldsymbol{p}) f_{k} f_{p}
$$


The mode coupling functional $\mathcal{F}_{q}$ is a quadratic expression in its variables $f_{k}$. It can be generalized by including higher order terms without complications. The coupling constants $V(\boldsymbol{q}, \boldsymbol{k}, \boldsymbol{p})$ are nonnegative symmetric functions of $\boldsymbol{k}, \boldsymbol{p}$, and are given in terms of the structure factor $S_{q}$.

The second kernel $\delta_{q}(z)$ is an additional backflow term which induces hopping over barriers. It is clear from (4) that this term will dominate the transport when the trapping mechanism in $m_{q}(z)$ becomes sufficiently large. For $\delta_{q}(z)$ one derives a similar mode-coupling expression as in (6), where now also longitudinal and transversal current correlation functions enter. Since currents always decay to zero for long times due to exchange of momentum between particles, $\delta_{q}(z)$ will exhibit a regular frequency variation for small $z$. We may therefore make the simplification $\delta_{q}(z) \approx \mathrm{i} \delta_{q}$, where [10]

$$
\delta_{q}=\int_{t_{B}}^{\infty} \mathrm{d} t \sum_{k, p} U(\boldsymbol{q}, \boldsymbol{k}, \boldsymbol{p}) \frac{\partial}{\partial t} \phi_{k}(t) \frac{\partial}{\partial t} \phi_{p}(t) .
$$

Here $t_{B}$ is a cutoff time which should be chosen sufficiently large to avoid contributions already included in the term $\left[\nu_{q}(z)+m_{q}(z)\right]$. The coupling constants $U(\boldsymbol{q}, \boldsymbol{k}, \boldsymbol{p})$ contain the contributions from the transverse currents, while the longitudinal currents have been expressed in terms of $(\partial / \partial t) \phi_{q}(t)$. The fluctuations which contribute to $(7)$ can be expressed in terms of the dynamical compressibility $\chi_{q}(t)=-\beta(\partial / \partial t) \phi_{q}(t)$, and may be interpreted as volume fluctuations. To allow for an atom to eventually escape from its surrounding cage such fluctuations must obviously be included. Through the contribution $m_{q}(z)$ in (5) and (6) one accounts only for an average cage around any atom built from the density of surrounding atoms.

The equations above represent a closed set of equations for the density propagator $\phi_{q}(t)$, which depend on the parameters $V(\boldsymbol{q}, \boldsymbol{k}, \boldsymbol{p})$ and $U(\boldsymbol{q}, \boldsymbol{k}, \boldsymbol{p})$. We will treat the wave vectors $q$, $k$, etc. as members of a finite set of labels $\left\{q_{1}, \ldots, q_{M}\right\}$, which implies that there is a finite number, say $N$, of coupling coefficients. They will be combined to the vectors $\boldsymbol{V} \in \mathcal{K}$ and $U \in \mathcal{K}^{\prime}$ in some parameter spaces $\mathcal{K}$ and $\mathcal{K}^{\prime}$.

In the supercooled liquid and near the glass transition the nonlinear feedback mechanism described above implies that $m_{q}(z)$ becomes very large for small frequencies. Compared with $m_{q}(z \approx 0)$ one can neglect the regular term $\left(z+\Omega_{q}^{2} \nu_{q}(z)\right)$ for small values of $z$. Then the relation (2) between correlator and kernel simplifies to

$$
\frac{\phi_{q}(z)}{1+\left[z+\mathrm{i} \delta_{q}\right] \phi_{q}(z)}=m_{q}(z)
$$

Equation (8) is the basis of all the asymptotic results on the glass transition singularities, both in the $\alpha$ and $\beta$-relaxation regions. The theory studies the long time properties of $\phi_{q}(t)$ or the low frequency behaviour of the spectra $\phi_{q}^{\prime \prime}(\omega)$ and corresponding susceptibilities $\chi_{q}^{\prime \prime}(\omega)$ in their dependence on the parameter vector $\boldsymbol{X}=(\boldsymbol{V}, \boldsymbol{U}) \in \mathcal{K} \times \mathcal{K}^{\prime}=\mathcal{R}$.

\section{Nonergodicity parameter}

With $\delta_{\boldsymbol{q}}=0$ in (8) one gets an ideal glass transition which is characterized by a change in dynamics from ergodic to nonergodic behaviour if the control parameter vector $\boldsymbol{V}$ crosses some hypersurface $S_{\mathrm{c}}$ in parameter space $\mathcal{K}$. On one side of $S_{\mathrm{c}}$ correlations decay to zero for large times, i.e. $\phi_{q}(t \rightarrow \infty)=0$. On the other side of $S_{c}$ correlations do not relax to zero for large times and $\phi_{q}(t \rightarrow \infty)=f_{q}>0$. The former case implies that an intial disturbance in the medium will decay with time and the system returns to the equilibrium state. In the latter case however, a disturbance will be arrested, and does not decay with time. Due to the strong interaction between the particles the system has become localized. We refer to this as an ideal glassy state, and it is characterized by 
the appearence of a nonergodicity pole $\phi_{q}(z) \rightarrow-f_{q} / z$ for $z \rightarrow 0$. The corresponding spectrum has an elastic line contribution $\phi_{q}^{\prime \prime}(\omega \approx 0)=\pi f_{q} \delta(\omega)$ in addition to a continuum. In the real case with $\delta_{q} \neq 0$ this elastic peak will be smeared due to hopping over barriers. There is no longer any strict nonergidicity pole for $z=0$. Nevertheless for $\delta_{q}$ sufficiently small, the underlying ideal transition will show up as a plateau region in $\phi_{q}(t)$, around the value $f_{q}$, corresponding to a metastable state, which can exist for a very long time. The corresponding elastic peak will be broadened to a quasielastic one, which is the structural relaxation or $\alpha$ peak. The nonergodicity parameter $f_{q}$ then appears as the area under this peak.

The parameter $f_{q}$ of the underlying ideal transition enter in an essential way even when $\delta_{q} \neq 0$. From (8) with $\delta_{q}=0$ it follows that $f_{q}(\boldsymbol{V})$ is a solution of

$$
f_{q} /\left(1-f_{q}\right)=\mathcal{F}_{q}\left(\boldsymbol{V}, f_{k}\right)
$$

We notice that $f_{q}$ depends on the parameters $\boldsymbol{V} \in \mathcal{K}$ defining the system. Let $f_{q}(\boldsymbol{V})$ denote the non-ergodicity parameter for point $\boldsymbol{V}$ provided it is non-zero. From (6) and (9) it is clear, that in general $f_{q}(\boldsymbol{V})>0$, unless $f_{q}=0$ for all $q$. Equation (9) may have other solutions besides $f_{q}=0$ and $f_{q}=f_{q}(\boldsymbol{V})$ [11]. It can be proved that the glass form factor $f_{q}(\boldsymbol{V})$ is the largest of all solutions of $(9)$, which may exist for a given $V$ [5].

We can also show that the space $\mathcal{K}$ has a strong coupling region $\mathcal{D}_{\infty}$, such that for $\boldsymbol{V} \in \mathcal{D}_{\infty}$ there is a non-zero glass form factor $f_{q}(\boldsymbol{V})$, which is analytic in $\boldsymbol{V}$ and behaves asymptotically like

$$
f_{q}(\boldsymbol{V})=1-\left[1 / \mathcal{F}_{q}(\boldsymbol{V}, 1)\right]+O\left(1 / \mathcal{F}_{q}(\boldsymbol{V}, 1)^{2}\right)
$$

Similarly it is possible to define a weak coupling region $\mathcal{D}_{0}$ in $\mathcal{K}$ where the only solution is $f_{q}(\boldsymbol{V})=0$ if $V \in \mathcal{D}_{0}$. Therefore the parameter space $\mathcal{K}$ splits into disjoint regions. The set of liquid states $\mathcal{D}_{L}$, which is the set of points $\boldsymbol{V}$ with $f_{q}(\boldsymbol{V})=0$. The set of ideal glassy states $\mathcal{D}_{G}$, which is the set of points $\boldsymbol{V}$ where $f_{q}(\boldsymbol{V})>0$, and the boundary $S_{c}$ separating $\mathcal{D}_{L}$ and $\mathcal{D}_{G}$. The points $\boldsymbol{V}_{c} \in S_{c}$, the set of glass transition singularities, are the singularities of function $f_{q}(\boldsymbol{V})$. They are those special bifurcation singularities of (9), which appear as limits of the correlation function. The liquid states contain the weak coupling region introduced above $\mathcal{D}_{L} \supset \mathcal{D}_{0}$. Similarly there is a strong coupling region of glass states $\mathcal{D}_{G} \supset \mathcal{D}_{\infty}$.

The coupling coefficients $V$ depends implicitly on temperature through the static correlation functions. When the temperature is changed the parameter point $\boldsymbol{V}(T)$ describes a curve $C$ in parameter space $\mathcal{K}$. This curve starts in $\mathcal{D}_{0}$ and runs to $\mathcal{D}_{\infty}$. So there must be a smallest $T$, to be denoted by $T_{\mathrm{c}}$, with the property: $\boldsymbol{V}(T) \in \mathcal{D}_{L}$ for $T>T_{c}$ and $\boldsymbol{V}\left(T_{\mathrm{c}}\right)=\boldsymbol{V}_{\mathrm{c}} \in S_{\mathrm{c}}$.

Let us consider a solution of (9) for a parameter point close to the critical one $V_{\mathrm{c}}$. All $M$ dimensional vectors $f_{q}$ are restricted by $0<f_{q}<1$. To analyze other solutions near the specified one, the parameter vector shall be measured relative to $\boldsymbol{V}_{\mathrm{c}}$

$$
V_{r}=V_{r}^{c}+v_{r}, \quad r=1, \ldots, N
$$

The deviation of $f_{q}$ from $f_{q}^{c}$ can be written in terms of a vector $g_{q}$ :

$$
f_{q}=f_{q}^{c}+\left(1-f_{q}^{c}\right)^{2} g_{q}, \quad q=1, \ldots, M .
$$

The mode coupling polynomial can be rearranged to one in $g_{q}$ by a straightforward Taylor expansion. The critical points $\boldsymbol{V}_{\mathrm{c}}$ appear for parameter points where one or more eigenvalues to the stability matrix $C_{q k}$ becomes one. Here $C_{q k}$ is given by

$$
C_{q k}\left(\boldsymbol{V}, f^{c}\right)=\left[\partial \mathcal{F}_{q}\left[\boldsymbol{V}, f_{k}^{c}\right] / \partial f_{k}^{c}\right]\left[1-f_{k}^{c}\right]^{2}
$$


From (6) it follows that $C_{q k}$ has only positive elements so there is a largest nondegenerate eigenvalue $E_{0}>0$, such that $\left|E_{i}\right|<E_{0}, i=1,2 \ldots$ [12]. The corresponding right and left eigenvectors $e_{q}$ and $\hat{e}_{q}$ can be chosen as positive and defined up to a constant. These eigenvectors define a singular direction in parameter space $\mathcal{K}$ and to leading order in the separation from the critical points we have $g_{q}=e_{q} g$, where the single parameter $g$ satisfies the equation:

$$
\delta_{0}+\delta_{1} g+\delta_{2} g^{2}+\delta_{3} g^{3}+\cdots=0
$$

The various coefficients $\delta_{k}$ are given by certain wave-vector integrals over the coupling constants in (6) [5].

Equation (14) generates the cuspoid family of singularities $A_{k}$ with $k \geq 2$ [13,14]. At the critical points $\boldsymbol{V}=\boldsymbol{V}_{\mathrm{c}}$ we must have $\delta_{0}^{c}=\delta_{1}^{\mathrm{c}}=0$. For parameter points such that $\delta_{2}^{\mathrm{c}} \neq 0$ we obtain an $A_{2}$ or fold singularity. If in addition $\delta_{2}^{\mathrm{c}}=0$ but $\delta_{3}^{\mathrm{c}} \neq 0$ we obtain an $A_{3}$ or cusp singularity and so on.

It is well known that (14) can be transformed into a canonical form for every cuspoid singularity. For the fold singularity we obtain

$$
\sigma-(1-\lambda) g^{2}=0
$$

where $\sigma=\delta_{0}\left(\boldsymbol{V}, f^{c}\right)$ is a canonical mathematical control parameter which we refer to as the separation parameter, and $\lambda=1+\delta_{2}<1$ is the exponent parameter which plays a crucial role for the dynamics. For the nonergodicity parameter this gives the singular behaviour

$$
f_{q}=f_{q}^{\mathrm{c}}+h_{q} \sqrt{\sigma /(1-\lambda)}, \quad \sigma \rightarrow+0 .
$$

and $f_{q}=0$ for $\sigma<0$. Here $h_{q}=\left(1-f_{q}^{c}\right)^{2} e_{q}$ is a critical amplitude. So near a fold singularity the nonergodicity parameter has a discontinous jump followed by a square root increase, and it depends essentially only on one relevant parameter, namely the mathematical separation parameter $\sigma$. The separation parameter $\sigma$ depends smoothly on the physical separation parameter $\epsilon=\left(T_{\mathrm{c}}-T\right) / T_{\mathrm{c}}$ and one finds $\sigma \propto \epsilon^{2}$ (type A) or $\sigma \propto \epsilon$ (type B). In the following only the case $\sigma \propto \epsilon$ will be considered. This implies that $\sigma<0$ for $T>T_{\mathrm{c}}$ and $\sigma>0$ for $T<T_{\mathrm{c}}$, and the transition point is located where $\sigma=0$.

Equation (16) can be generalized straightforwardly to the general cuspoid $A_{k}$ which has a canonical representation with $k-1$ relevant parameters $\delta_{0}, \ldots, \delta_{k-2}[5]$, but these results are not needed here.

\section{4. $\beta$-relaxation process}

The $\beta$-process exists on mesoscopic time-scales between the microscopic and $\alpha$-regions. This region is characterized by a plateau region in $\phi_{q}(t)$ around the value $f_{q}$ provided the parameter point $\boldsymbol{X}$ is sufficiently close to a singularity $\boldsymbol{V}_{\mathrm{c}}$. In addition that the separation parameter $\sigma$ is sufficiently small this also requires that $\delta_{q}$ is small in some sense. In analogy to (12) we may introduce a more regular function $G_{q}(t)$ as

$$
\phi_{q}(t)=f_{q}^{c}+\left[1-f_{q}^{c}\right]^{2} G_{q}(t)
$$

Following arguments which lead to (14) we find that in leading order $G_{q}(t)=e_{q} G(t)$ [15], and therefore

$$
\phi_{q}(t)=f_{q}^{c}+h_{q} G(t)
$$

valid in the time region $t_{0} \ll t \ll \tau_{\alpha}$, where $t_{0}$ is a microscopic time. The whole relaxation pattern in the $\beta$-region is therefore described by one single function $G(t)$ only. Similar relations hold for correlation functions $\phi_{X Y}(q t)$ of any variables $X$ and $Y$ with a nonzero overlap with density 
fluctuations. For instance with $X$ and $Y$ representing the microscopic dipole-moment we find for the dielectric function [16]

$$
\epsilon(z)=f_{\epsilon}+h_{\epsilon} z G(z)
$$

Similar result holds for other susceptibilities.

The function $G(t)$ satisfies the equation $[9,17,18]$

$$
\begin{aligned}
& -\delta_{0} / z+\mathrm{i} \delta / z^{2}+\delta_{1} G(z)+z G^{2}(z)+\left(1+\delta_{2}\right) L T\left[G^{2}(t)\right](z) \\
& +\left[\delta_{3}+\gamma_{3}\right] L T\left[G^{3}(t)\right](z)-\gamma_{3} z^{2} G^{3}(z)+\cdots=0,
\end{aligned}
$$

The parameters $\delta_{k}$ were introduced above, and $\gamma_{k}$ are also given by certain wave-vector integrals. Similarly $\delta$ is given by an integral over $\delta_{q}$. This equation has a structure similar to the static one in (14). The latter is in fact just a special case of (20) if we take $\delta=0$ and look for solutions $G(z)=-g / z$. Due to a $\delta \neq 0$ the solutions to (20) contain no constant part or $1 / z$ pole, but will always decay.

Near the bifurcation points $\boldsymbol{V}_{\mathbf{c}}$ however, there is an additional subtle low frequency singularity in $G(z)$, apart from any $1 / z$-pole. This singularity depends sensitively on the parameters $\delta, \delta_{0}, \delta_{1}, \delta_{2}$ etc., and can therefore be classified according to the corresponding cuspoid $A_{k}$. In the present paper we will only consider the fold singularity $A_{2}$ which is completely specified by the separation parameter $\sigma=\delta_{0}$, the hopping rate $\delta$ and the exponent parameter $\lambda=1-\delta_{2}$, while cubic and higher order terms in (20) only gives small corrections to the leading order results.

\subsection{Critical spectrum}

Of particular interset is the solution of (20) at the critical points $\boldsymbol{X}_{\mathbf{c}}=\left(\boldsymbol{V}_{\mathrm{c}}, \mathbf{0}\right)$. This implies $\delta_{0}=\delta=\delta_{1}=0$ in $(20)$, while $\delta_{2} \neq 0$ or $\lambda<1$. The equation for the critical spectrum reduces to $z G^{2}(z)+\lambda L T\left[G^{2}(t)\right](z)=0$. This equation is solved by a power law

$$
G(t)=\left(t_{0} / t\right)^{a}
$$

where $t_{0}$ must be determined by matching to the microscopic region. The exponent $a$ is determined from the exponent parameter through the equation

$$
\lambda=\Gamma^{2}(1-a) / \Gamma(1-2 a)
$$

A positive spectrum requires $0<a<1 / 2$. A second solution $b=-a$ to (22) with $0<b<1$ also exist and appears below.

\subsection{Scaling law}

For parameter points $\boldsymbol{X}$ close to the transition surface $S_{\mathrm{c}}$ the function $G(t)$ is given by various scaling laws. Near a fold singularity we find the solution in terms of a two parameter scaling law $[9,19]$

$$
G(t)=c_{\Omega} g(\hat{t}, \hat{\sigma}, \hat{\delta}) .
$$

where $\hat{t}=t \Omega / t_{0}, \hat{\sigma}=\sigma / \Omega^{2 a}$ and $\hat{\delta}=\delta t_{0} / \Omega^{1+2 a}$ are rescaled variables. The solution depends, through the correlation scale $c_{\Omega}=\Omega^{a}$ and the dimensionless frequency scale $\Omega$, crucially on 
the distance from the transition point which is reached along a scaling line when $\Omega \rightarrow 0$. The masterfunctions $g$ satisfy the equation [19]

$$
\hat{\sigma}-\hat{\delta} \hat{t}+\lambda g^{2}(\hat{t})=\frac{\mathrm{d}}{\mathrm{d} \hat{t}} \int_{0}^{\hat{t}} \mathrm{~d} \hat{t}^{\prime} g\left(\hat{t}-\hat{t}^{\prime}\right) g\left(\hat{t}^{\prime}\right),
$$

The function $g$ is determined by the equilibrium structure, which enter in (23b) only through the parameter $\lambda$. The hopping rate $\delta$ introduces a natural scale for the separation parameter [9]:

$$
\sigma_{0}=\left(\delta t_{0}\right)^{2 a /(1+2 a)}
$$

The three regimes $\sigma \ll-\sigma_{0},|\sigma| \ll \sigma_{0}$ and $\sigma \gg \sigma_{0}$ exhibit spectra, which are qualitative quite different [19]. These details will not be considered here, but we will restrict our attention to the ideal transition.

The ideal glass transition is obtained with $\delta=0$ in (23b). In this case one can choose $\Omega=|\sigma|^{1 / 2 a}$, i.e $\hat{\sigma}= \pm 1$, and with $g_{ \pm}(\hat{t})=g(\hat{t}, \pm 1,0)$ one gets a conventional scaling law

$$
G(t)=c_{\sigma} g_{ \pm}\left(t / t_{\sigma}\right)
$$

Here \pm refers to $\sigma \gtrless 0$ respectively. The correlation scale becomes $c_{\sigma}=\sqrt{|\sigma|}$ and the $\beta$-relaxation time scale is $t_{\sigma}=t_{0} /|\sigma|^{1 / 2 a}$. The scaling functions $g_{ \pm}$can be obtained for any value of $\lambda$ [20]. For short times we obtain the critical decay in $(21), g_{ \pm}(\hat{t} \ll 1)=1 / \hat{t}^{a}$. For long rescaled times one can also find explicit expressions

$$
\begin{gathered}
g_{+}(\hat{t} \gg 1)=1 / \sqrt{1-\lambda}, \\
g_{-}(\hat{t} \gg 1)=-B \tau^{b}
\end{gathered}
$$

where $B>0$. The exponents $b$ are given by the second solution to (22) with $0<b<1$. Equation (25b) gives the von Schweidler law

$$
\phi_{q}(t)-f_{q}^{\mathrm{c}}=-h_{q} B|\sigma|^{1 / 2}\left(t / t_{\sigma}\right)^{b}=-h_{q} B\left(t / t_{\sigma}^{\prime}\right)^{b} .
$$

which also describes the initial decay of the $\alpha$-process. The new time scale $t_{\sigma}^{\prime}=t_{\sigma} /|\sigma|^{1 / 2 b}$ is the predicted scale for the $\alpha$-relaxation process, which will be discussed more fully in next section.

For the susceptibility or the dielectric loss these results give the scaling function

$$
\chi^{\prime \prime}(\omega)=h_{q}|\sigma|^{1 / 2} \hat{\chi}_{ \pm}^{\prime \prime}\left(\omega / \omega_{\sigma}\right)
$$

where $\omega_{\sigma} \propto 1 / t_{\sigma}$. In the liquid state with $\sigma<0$ the critical decay in (21) implies a decrease of $\chi^{\prime \prime}(\omega) \propto \omega^{a}$ with decreasing frequency, while the von Schweidler law (26) implies a subsequent increase $\chi_{-}^{\prime \prime}(\omega) \propto \omega^{-b}$. Hence for $T>T_{\mathrm{c}}$ the $\beta$-decay process can be detected as a minimum in the susceptibility $\chi_{-}^{\prime \prime}(\omega)$ located above the $\alpha$-peak at some frequency $\omega_{\min }$. The scaling law in (27) implies that the position of the minimum $\omega_{\min }$, and the value of $\chi_{-}^{\prime \prime}$ at the minimum $\chi_{\min }^{\prime \prime}=\chi_{-}^{\prime \prime}\left(\omega_{\min }\right)$ are described by

$$
\begin{gathered}
\chi_{\min }^{\prime \prime} \propto c_{\sigma}=|\sigma|^{1 / 2}, \\
\omega_{\min } \propto \omega_{\sigma} \propto|\sigma|^{1 / 2 a} .
\end{gathered}
$$

In the ideal glass state below $T_{c}$ the susceptibility is given by

$$
\begin{aligned}
& \chi_{+}^{\prime \prime}(\omega) \propto h_{q}|\sigma|^{1 / 2}\left(\omega / \omega_{\sigma}\right) \quad 0<\left(\omega / \omega_{\sigma}\right) \ll 1, \\
& \chi_{+}^{\prime \prime}(\omega) \propto h_{q} B|\sigma|^{1 / 2}\left(\omega / \omega_{\sigma}\right)^{a} \quad 1 \ll\left(\omega / \omega_{\sigma}\right) \ll\left(\Omega_{q} / \omega_{\sigma}\right)
\end{aligned}
$$



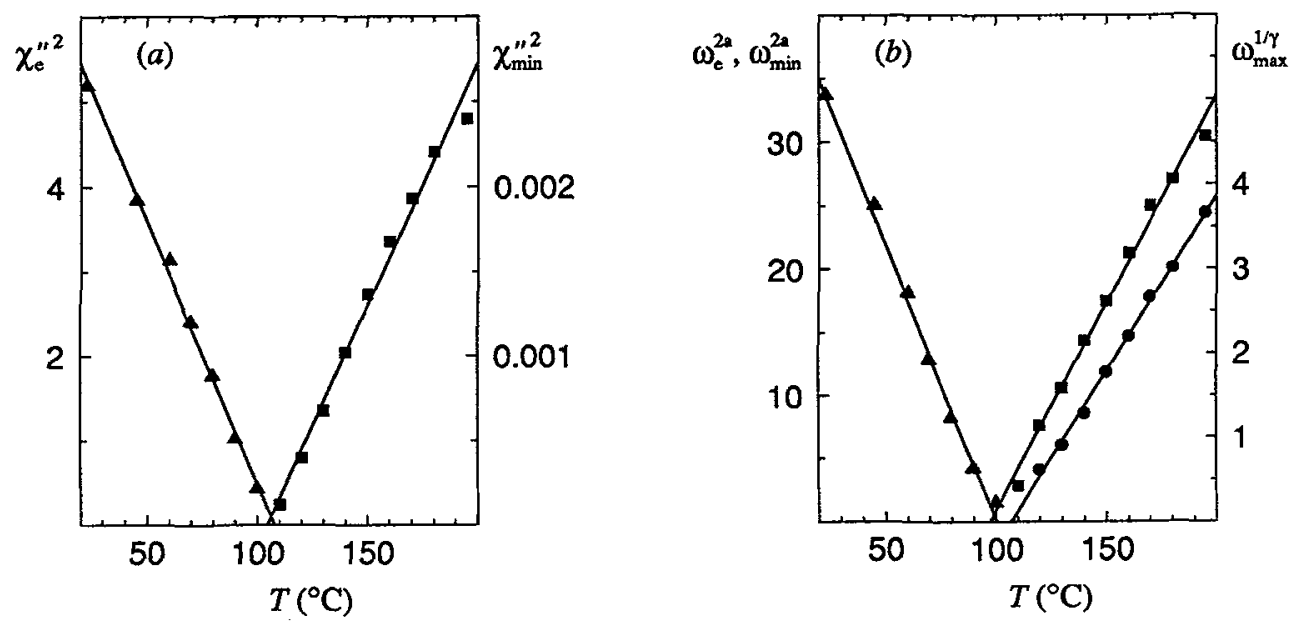

Figure 1. (a) Experimental values from [21] of $\chi_{\min }^{\prime \prime}{ }^{2}$ (squares, right scale) and $\chi_{\mathrm{e}}^{\prime \prime 2} \times 10^{5}$ (triangles, left scale) plotted versus temperature $T$. The straight lines are the best fit through the data points, and indicate the theoretical prediction in (28a) and (30a). The intersection with the abscissa gives the critical temperature $T_{c}$. (b) Squares show $\omega_{\min }^{2 a}$ (left scale), triangles show $\omega_{\mathrm{e}}^{2 a}$ (left scale) and circles $\omega_{\max }^{1 / \gamma}$ (right scale) versus temperature as obtained from experimental data. The full lines are the best fits through the respective data points, and gives the theoretical predictions in (28b) (30b) and (31). The intersection gives again the critical temperature $T_{c}$.

Therefore in the ideal glass state there exists a crossover for $\chi_{+}^{\prime \prime}(\omega)$ from white noise $\chi_{q}^{\prime \prime} \propto \omega$ to a fractal behaviour $\chi_{q}^{\prime \prime} \propto \omega^{a}$. If the crossover point is denoted as $\omega_{e}$ and $\chi_{e}^{\prime \prime}=\chi_{+}^{\prime \prime}\left(\omega_{e}\right)$, we find

$$
\begin{aligned}
& \chi_{e}^{\prime \prime} \propto|\sigma|^{1 / 2}, \\
& \omega_{\mathrm{e}} \propto \omega_{\sigma} \propto|\sigma|^{1 / 2 a} .
\end{aligned}
$$

From (26) we also find the predicted variation of the positions of the $\alpha$-peak maximum, $\omega_{\max }$

$$
\omega_{\max } \propto 1 / t_{\sigma}^{\prime} \propto|\sigma|^{\gamma} \quad \gamma=1 / 2 a+1 / 2 b .
$$

Recently susceptibility spectra for the molten salt $\mathrm{Ca}_{0.4} \mathrm{~K}_{0.6}\left(\mathrm{NO}_{3}\right)_{1.4}$ were obtained through light scattering by Cummins and coworkers [21]. These results exhibits the complete dynamical pattern with a microscopic band around $4 \mathrm{THz}$, an $\alpha$-peak which rapidly moves down in frequency with decreasing temperature, and the $\beta$-spectrum in between. These results are also the first where properties both abow and below $T_{\mathrm{c}}$ could be tested. The results for the various amplitudes and frequencies are shown in figures $1 \mathrm{a}$ and $1 \mathrm{~b}$ respectively. In figure $1 \mathrm{a}$ we show $\chi_{\min }^{\prime \prime}{ }^{2}$ (squares) and $\chi_{\mathrm{e}}^{\prime \prime 2}$ (triangles) versus temperature $T$. According to (28a) and (30a) the data should fall on straight lines, which is approximately the case as shown by the full lines. Similarly we show in figure $1 \mathrm{~b}$ $\omega_{\min }^{2 a}$ (squares), $\omega_{\mathrm{e}}^{2 a}$ (triangles) and $\omega_{\max }^{1 / \gamma}$ (circles) versus $T$. The data fall again on straight lines indicated by the full lines, and this supports the results in (28b) (30b) and (31). All data are consistent with a critical temperature $T_{\mathrm{c}} \approx 105^{\circ} \mathrm{C}$. Previous dielectric loss measurements on the polymeric systems polyethylene therephthalate (PET) and polyethylene oxybenzoate (PEOB) [22] were also sucessfully analysed within the MCT $[16,23]$.

The scaling functions $g_{ \pm}(\hat{t})$ can be calculated directly from (23b) and the results can be compared with photon correlation measurements, neutron spin echo measurements and molecular 
dynamics simulations. A comparison with light scattering measurements on a hard sphere colloidal system [24] gave a quantitative agreement between experiments and theory [25, 26]. There are also extensive molecular dynamics results on simple two component systems [27]. A similar analysis for three correlators describing relaxation with wave vector at the structure factor peak [28] produced reasonable agreement between data and MCT within the relevant intermediate time window [26].

\section{5. $\alpha$-relaxation process}

An understanding of the $\alpha$-peaks, i.e. an explanation of the temperature variation of the scale $\tau_{\alpha}$, of the $\alpha$-peak strength and, in general, of the $\alpha$-peak shape is essential for the explanation of the liquid to glass transition. From the basic equations (5-8) properties of the $\alpha$-relaxation process can be extracted. In this section the various results will be presented.

\subsection{Scaling behaviour}

With $\delta_{q}=0$ equation (8) is scale invariant, i.e. if $\phi_{q}$ is a solution, the same holds for the correlators with rescaled times $\phi_{q}^{y}$, where

$$
\phi_{q}^{y}(t)=\phi_{q}(t y), \quad \phi_{q}^{y}(z)=\phi_{q}(z / y) / y,
$$

with any $y>0$. With a finite $\delta_{q}$ this scale-invariance is lost since the hopping rate $\delta_{q}$ itself determines the scale for sufficiently long times or small frequencies. In any case the solution to (8) in the $\alpha$-relaxation region is given by a scaling law:

$$
\phi_{q}(t)=f_{q} F_{q}\left(t / \tau_{\alpha}\right)
$$

Here the scaling function $F$ is independent of temperature in leading order. The initial value $f_{q}$ determines the $\alpha$-peak area. The temperature dependence of $f_{q}$ is given in (16) for $T<T_{c}$, while for $T>T_{\mathrm{c}}$ one finds that the area is fixed at the constant value $f_{q}^{c}$. These predictions by the theory have been observed experimentally in several systems by neutron scattering techniques. The behaviour in (16) has been found in simple organic glass formers [29], in polymeric systems [30] and a molten salt [31].

The shape of $F_{q}(t)$ is not known precisely, but numerical solutions for various models have shown that it is very well approximated by a Kohlrausch law $F_{q}(t) \approx \exp \left(-t^{\beta}\right)$. The well known stretching phenomena of the $\alpha$-precess is therefore contained in the nonlinear equation (8). Some polymers exhibit a splitting of the low frequency susceptibility spectra into $\alpha^{\prime}-\alpha$-peak pairs. The whole spectrum follows usually the scaling law (33) and therefore both peaks have to be considered as parts of one and the same $\alpha$-process. Such double-peak patterns can indeed be obtained as generic results of the MCT. They occur if $\boldsymbol{V}$ is close to corners of the bifurcation hypersurface, which arises from self crossings of parts of $S_{c}$ [32]. These double peak structure show that the shape of $F_{q}(t)$ cannot in general be parametrized by a simple formula.

The origin of the stretching in the scaling function $F_{q}$ can be understood from the short time expansion of (8), i.e. for times $t \leq \tau_{\alpha}$. In this region (8) can be solved analytically, and the result is given by the von Schweidler law [5]

$$
\phi_{q}(t)=f_{q}-h_{q} B(\hat{t})^{b}+O\left(1 / \hat{t}^{b}\right)
$$

where $\hat{t}=t / \tau_{\alpha}$. The exponent $b$ is the same as in (26). For the ideal transition the von Schweidler law holds only for $T>T_{\mathrm{c}}$, where it signals the instability of the localized structure. For $T<T_{\mathrm{c}}$ 


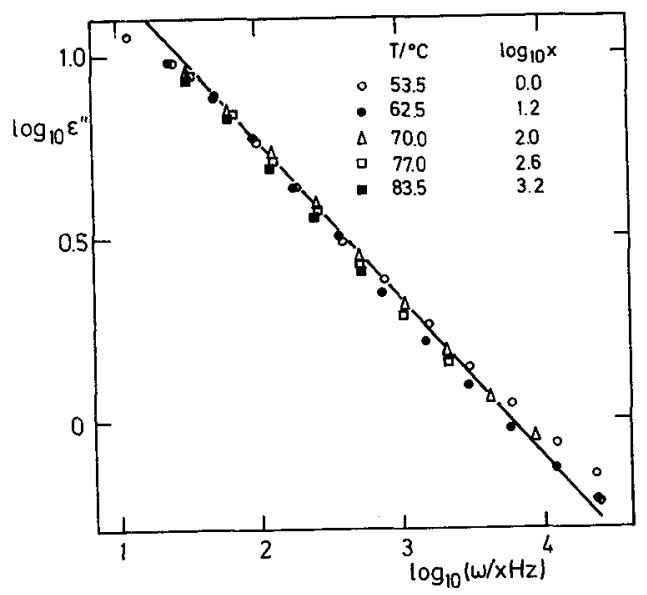

Figure 2. The high frequency wing of dielectric loss data for polyvinylacetat taken from Ishida et al [33]. The full line has slope 0.40

the function $\phi_{q}(t)$ would stay at the initial value $f_{q}$. The effect of a nonzero $\delta_{q}$ is to extend the validity of (33) to temperatures $T<T_{\mathrm{c}}$, and this explains why stretching in real systems is seen down to $T_{\mathrm{g}}$.

The von Schweidler behaviour in (34) can be observed in the high frequency wing of the $\alpha$-peak. This is illusteated in Fig. 2 for dielectric loss data obtained by Ishida et al [33] for polyvinylacetat. With the susceptibility $\chi_{q}^{\prime \prime}(\omega) \propto \omega \phi_{q}^{\prime \prime}(\omega)$ rescaled according to (33) the behaviour in (34) implies a $1 / \omega^{b}$ behaviour, which on a $\log -\log$ plot gives a straight line. This behaviour is clearly seen in Fig. 2 for a frequency interval of three decades. Jonscher has shown similar plots for many other disordered systems where the exponent $b$ range from 0.2 to 0.8 [34].

\section{2. $\alpha$-relaxation scale}

It follows from (26) that the von Schweidler law in (34) describes the long time dynamics for the $\beta$-relaxation with scale $t_{\sigma}$ as well as the short time dynamics for the $\alpha$-region with scale $\tau_{\alpha}$. The von Schweidler region describes the overlap between these two processes. The scale $\tau_{\alpha}$ in (34) can therefore be obtained by matching the two processes in the relevant time window. In the ideal case with $\delta_{q}=0$ this gives an algebraic divergence of $\tau_{\alpha}$

$$
\tau_{\alpha}=t_{0} /|\sigma|^{\gamma}
$$

We notice that as $T \rightarrow T_{\mathrm{c}} \tau_{\alpha} / t_{\sigma} \rightarrow \infty$. So the overlapping von Schweidler region defined by $t_{\sigma} \ll t \ll \tau_{\alpha}$ expands when approaching $T_{\mathrm{c}}$. We notice that the scale $\tau_{\alpha}$ depends on the two exponents $a$ and $b$. The former is related to the critical decay for $t \ll t_{\sigma}$ in (21). So there exist an intimate and nontrivial relation between the relaxation law for the $\beta$-process and the scale for $\alpha$-relaxation. The theory also predicts the relaxation time for all $\alpha$-processes to be proportional to $\tau_{\alpha}$ for $T>T_{c}$, i.e. there is an $\alpha$-scale universality in this temperature region. In particular there should be an algebraic variation of the viscosity $\eta \propto 1 /|\sigma|^{\gamma}$. This prediction was tested by Taborek et al for many different glassformers [35]. Their results showed that $\eta$ increased as in (35) up 
to viscosities around $100-1000$ poise, whereafter there was a crossover to a different behaviour. From this observation they anticipated the existence of a crossover temperature $T_{c}$ in the liquid.

The most important effect of the coupling to the currents, which introduce a $\delta_{q} \neq 0$, is the elimination of the divergency in (35) for $T \rightarrow T_{\mathrm{c}}+$. It also changes an associated ideal elastic line for $T<T_{\mathrm{c}}$ into a quasielastic $\alpha$-peak. As in the ideal case the scale $\tau_{\alpha}$ near and below $T_{\mathrm{c}}$ is determined by the dynamics of the $\beta$-process. There appears now an interplay between the motion on microscopic wave-lengths and the long wave-length motion. As a result one gets [10]

$$
\tau_{\alpha}=\tau_{0} \exp \left(E / k_{B} T\right)
$$

This implies an activated behaviour for the viscosity. The activation energy $E$ is predicted to depend on the compressibility of the system $E \propto 1 / \kappa_{T}$, which induces a slight temperature dependence.

\section{Conclusions}

The mode-coupling equations (5-8) represent a set of closed nonlinear equations for the density correlation function $\phi_{q}(t)$, which describe the structural relaxations in a supercooled liquid or a glass near the glass transition. Many features of the self-consistent solution of these equations are in general agreement with those found in real systems near the glass transition. Results for higher order cuspoids has also been obtained [18], and the results has sucessfully been compared with experimental results on polymeric systems $[16,36]$. In this case the susceptibility spectra can be obtained from properties of elliptic functions.

\section{References}

1 G. P. Johari and M. Goldstein J. Chem. Phys. 53 (1970) 2372; 55 (1971) 4245

2 G. P. Johari J. Chem. Phys. 58 (1973) 1766

3 E. Leutheusser Phys. Rev. A 29 (1984) 2765

4 U. Bengtzelius, W. Götze and A. Sjölander J. Phys. C: Solid State Phys. 17 (1984) 5915 U. Bengtzelius Phys. Rev. A 33 (1986) 3433; A 34 (1986) 5059

5 W. Götze Liquids, Freezing and Glass Transition ed. J. P. Hansen et al (North Holland, Amsterdam, 1991), p. 287.

6 W. Götze and L. Sjögren Rep. Prog. Phys. 55 (1992) 241

7 S. P. Das, G. F. Mazenko, S. Ramaswamy and J.J. Toner Phys. Rev. Lett. 54 (1985) 118

S.P. Das and G.F. Mazenko Phys. Rev. A 34 (1986) 2265

S.P. Das Phys. Rev. A 36 (1987) 211

8 L. Sjögren and A. Sjölander J. Phys. C: Solid State Phys. 12 (1979) 4369

L. Sjögren Phys. Rev. A 22 (1980) 2866

9 W. Götze and L. Sjögren Z. Phys. B 65 (1987) 415; J. Phys. C: Solid State Phys. 21 (1988) 3407

10 L. Sjögren Z. Phys. B 79 (1990) 5

11 W. Götze and R. Haussmann Z. Phys. B 72 (1988) 403

12 R. Bellman Introduction to Matrix Analysis (McGraw-Hill, New York, 1970)

13 A. Arnold Catastrophe Theory 2nd edn. (Springer, Heidelberg, 1976)

14 R. Gilmore Catastrophe Theory for Scientists and Engineers (Wiley, New York, 1981)

15 W. Götze Z. Phys. B 60 (1985) 195; in Amorphous and Liquid Materials ed E. Lüscher et al (Dordrecht: Martinus Nijhoff) (1987) p. 34

16 L. Sjögren J. Phys: Condensed Matter 3 (1991) 5023 


\section{B. Frick and D. Richter
Springer) (1989) p. 38}

B. Frick, D. Richter, W. Petry and U. Buchenau Z. Phys. B 70 (1988) 73

B. Frick, D. Richter and C. Ritter Euro Phys. Lett. 9 (1989) 557

B. Frick, B. Farago and D. Richter Phys. Rev. Lett. 64 (1990) 2921

D. Richter, R. Zorn, B. Frick and B. Farago Ber. Bunsenges. Phys. Chem. 95 (1991) 1111

31 F. Mezei, W. Knaak and B. Farago Phys. Rev. Lett. 58 (1987) 571; Phys. Scr. T 19 (1987) 363

F. Mezei Dynamics of Disordered Materials ed D. Richter et al (Berlin: Springer) (1989) p. 164

F. Mezei Ber. Bunsenges. Phys. Chem. 95 (1991) 1118

32 M. Fuchs, W. Götze, I. Hofacker and A. Latz J. Phys: Condensed Matter 149 (1991) 5047

33 Y. Ishida, M. Matsuo and K. Yamafuji Koll. Z. Z. Polym. 180 (1962) 108

34 A. K. Jonscher Nature 267 (1977) 673

35 P. Taborek, R.N. Kleinman and D.J. Bishop Phys. Rev. B 34 (1986) 1835

36 S. Flach, W. Götze and L. Sjögren Z. Phys. B 87 (1992) 29 